\title{
An Improved Method for Tracing IP Packet's Source
}

\author{
Chandrasekar Ravi", P. S. Ramesh and N. Deepa \\ School of Information Technology and Engineering,VIT University, Vellore, \\ Tamil Nadu - 632 014, India; chandrasekar.r@vit.ac.in
}

\begin{abstract}
IP spoofing and Denial of Service (DoS) has become the most important security threat faced by the Internet community today. One of the effective methods of defending against the types of attack is to efficiently locate its source(s). To locate the packet source, a 16-bit hybrid trace back method has been proposed. It uses both the marking and logging methods along with efficient storage method to achieve zero false positive and zero false negative rates. Furthermore, the evaluation of the proposed method on the basis of efficiency of trace back shows that it performs much better than the existing method.
\end{abstract}

Keywords: Denial of Service (DoS), Hash Table, IP Spoofing, IP Trace Back

\section{Introduction}

A great deal of emphasis has been given to deal with IP spoofing and DoS attacks by many researchers all over the world. But still there are no efficient and effective methods to eliminate or discover these types of attack. DoS ${ }^{1}$ attacks causes consumption of resources related with various network elements like web services, routers, firewalls, end-hosts etc., which impedes the efficient functioning of the service in accordance with their intended purpose. This impacts not only the Quality of Service (QoS) but also affects the availability of services. The other one is IP spoofing ${ }^{2}$ in which network packets are created with forged IP address with the purpose of concealing the identity of the sender or impersonating another computer system. For these reasons, developing an effective mechanism to locate the source/origin of the packet has become an important issue now-a-days. Several solutions have been proposed which can be broadly divided into the following mechanisms: packet marking, packet logging and hybrid mechanism. In the packet marking mechanism, all the packets on the way to destination gets marked i.e. it stores some information about the routers through which it has passed inside them based on the algorithm ${ }^{3}$. Probabilistic packet marking and determin- istic packet marking are some of the examples for packet marking mechanism. In packet logging mechanism ${ }^{4}$, the information about the packets is stored in the routers as the packets pass through them. Later, this information is used together to reconstruct the path to trace the origin. In the hybrid trace back mechanism, both the packet marking and packet logging methods are used together to provide more efficient way to trace back the origin. The packet logging and the packet marking mechanism are easy to implement and use, but they fail to provide an effective trace back method i.e. zero false positive and zero false negative rate in tracing back the source. The hybrid trace back mechanism provides more effective way to trace back the source than the marking and logging methods, but does not provide zero false negative and zero false positive rates and it also suffers from the problem of high storage requirements. To overcome the above limitations, a new hybrid trace back method has been proposed, that marks the router interface number and integrates packet logging with the hash table. The proposed trace back method is designed to achieve the following properties:minimal logging of packets and the use of hash table, zero false positive and false negative rate and higher efficiency in path reconstruction. The rest of the paper is organized as follows. Section II discusses

${ }^{*}$ Author for correspondence 
the literature survey. Section III introduces the proposed work. Experimental setup is discussed in Section IV. Experimental results are analyzed and compared in Section V. Section VI concludes the paper.

\section{Related Works}

There are many IP trace back methods proposed by many researchers which uses the basic traceback techniques i.e. packet marking and/or packet logging. For instance, Belenky and Ansari proposed a technique ${ }^{5}$ where the marking of the egress router information is done on the identification and flag fields. However, it does not provide a zero false negative and false positive rates. Another technique ${ }^{6}$ proposed by Snoeren et al., converts the unchanged part of a packet into digest and is stored using bloom filter, but it suffers from false positive problem and the requirement of large storage space. Gong and Sarc proposed a Hybrid IP Trace back method ${ }^{7}$, called HIT, which uses both packet marking and packet logging method. Malliga and Tamilarasi proposed a new trace back method, called $\mathrm{MORE}^{8}$, where a 32-bit marking field is used to mark the packet. They used mathematical models to mark the fields. Even though the above method performs more efficient than Huffman code ${ }^{9}$, it suffers from various demerits such as firstly high false positive rate when collision occurs in a log table, secondly an inefficient search of the log table if the table is big and finally the trace back will fail if the marking field is zero even after logging. Yang and Ming-Chien-Yang proposed a technique ${ }^{10}$ called RIHT where 8-bit marking field is used and for the logging purposes hash table is used. However the number of times a packet logging occurs increases if the network traffic increases. Due to the above problems, a new 16-bit hybrid IP trace back method has been proposed in this paper, which will greatly reduce the number of occurrence of packet logging.

\section{Proposed Method}

In this proposed method, the interface numbers of the routers are marked on the packet's 16 bit marking field as they travel from source to destination. As the size of the marking field is limited, the hash table has to be used to $\log$ the marking field and store the index of the hash table on the packet. This mechanism continues till the packet reaches the destination. At the destination, the path can be easily traced back by reversing the entire process.
The example network, shown in Figure 1, contains many routers, a source and a destination. The border router (r1) receives the packet from the source and it forwards the packet to the upstream router. The following assumptions ${ }^{10}$ about the network, to be experimented, are considered: Firstly, each router will have specific values for its entire interface and it should be made known to its neighboring router. Secondly, the router can differentiate the packets coming from a neighboring router or a local network. Thirdly, the trace back method is implemented on each and every router. Finally, the network topology cannot be changed frequently.

As shown in Figure 2, whenever a packet is received at the core router initially, the pmark value is calculated

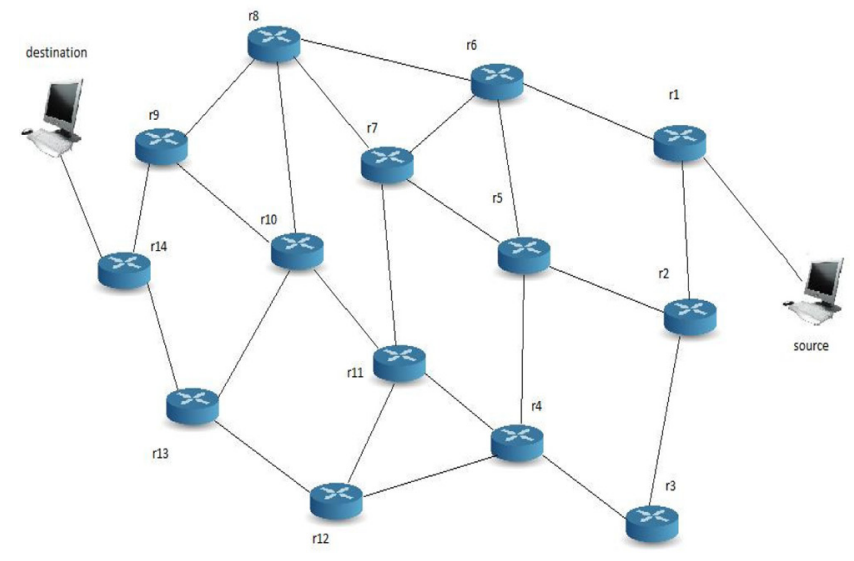

Figure 1. An example network.

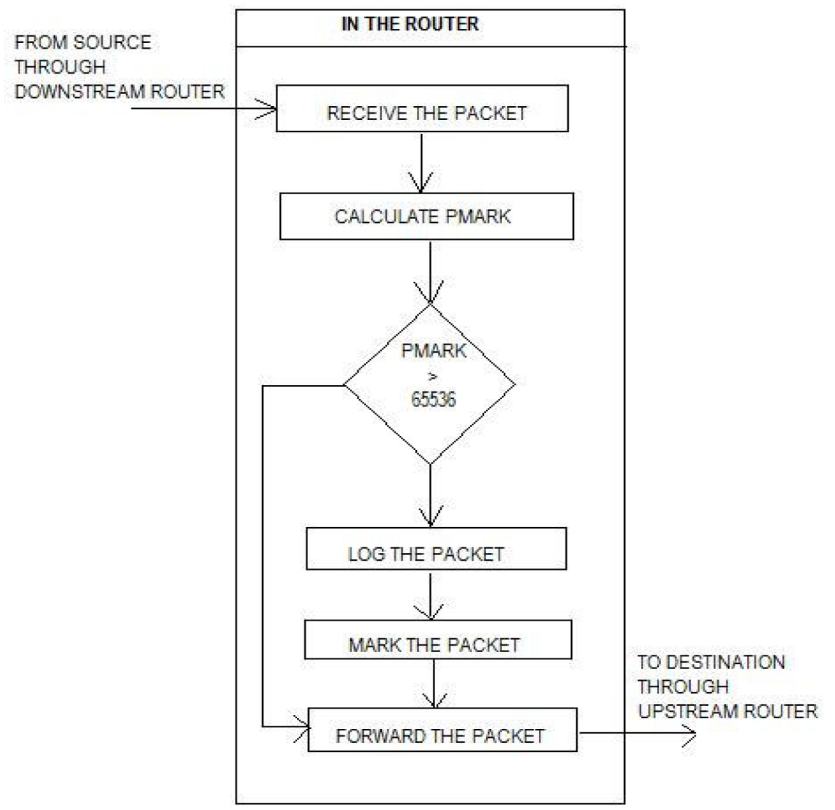

Figure 2. Architecture of the proposed IP trace back method. 
then if the pmark value is greater than 216 it is logged in the hash table and then forwarded; else the packet is forwarded with the new pmark value.

The pmark value present in the packet along with the interface number is stored in the hash table. To calculate the hash value, Pearsons Hashing is used. It is a non-cryptographic hash function which produces only a single byte output for inputs of any number of bytes. It is simple and executes quickly as it contains less number of instructions. There is no simple class of input for which collision can occur. To mark the packet, the flag field and the fragmentation field are used, as shown in Figure 3. According to Stocia et al. ${ }^{11}$ and John et $\mathrm{al}^{12}$, the chances of a packet being segmented has come down from $0.25 \%$ to $0.06 \%$. It has been determined that around $60 \%$ of the fragmented packets are attacking packets ${ }^{13}$. Thus overwriting these two fields will not affect most legitimate packets.

When a border router receives a packet from the source or a local network, it marks the marking field with zero and forwards the packet to the upstream router. When the core router receives the packet, it computes the new pnewmark $=$ pmark $^{*}$ (no._of_interface $\left.)+1\right)+($ value of_the_interface) +1 . If the pnewmark is not over flow i.e. not greater than 62,536 , then it replaces pmark with the pnewmark value and forwards it to the next upstream router. If the pnewmark is an over flow then it computes the index $=\mathrm{H}$ (pmark). If the index is equal to zero or not

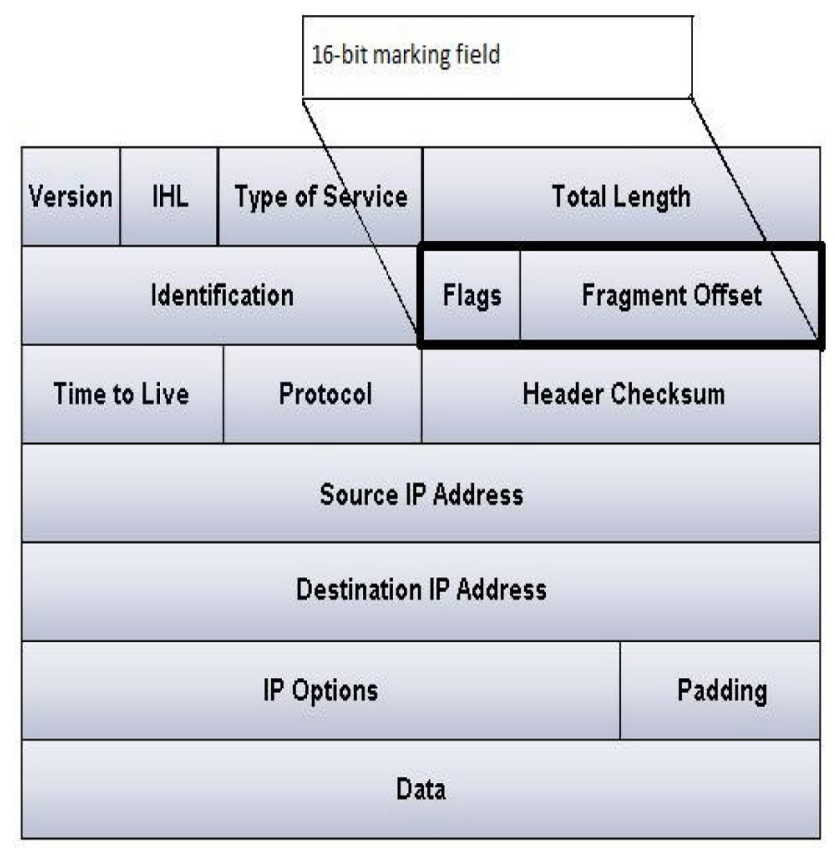

Figure 3. Showing the fields used for marking the packet. empty or equal to the pmark value then a quadratic probing algorithm is used to compute the new index. After getting the index it stores the pmark and the UI value at the index and computes the pnewmark $=$ index ${ }^{\star}$ (no._of_ interface)+1). This pnewmark value is then stored in the packet and forwarded to the next upstream router. This process continues until the packet reaches the destination. The path reconstruction ${ }^{10}$ takes place by reversing the entire process.

\section{Experimental Setup}

For the simulation of the proposed 16-bit IP trace back method, OMNET++ is used as the simulation software. It is a discrete event simulation environment primarily used simulation in the field of communication network.

Here the efficiency $(€)$ is calculated by:

$\frac{\text { number of times packet not been logged }}{\text { max imum number of timea packet can be logged }} \times 100$

where, the maximum number of time, a packet passes through a router, is $15^{11}$.

\section{Result and Analysis}

The efficiency of the proposed method for 8-bit and 16-bit marking field is calculated, tabulated and analyzed. It is observed that, the number of times a packet is logged in a 8-bit marking field is much more as compared to 16-bit marking field.

From Table 1 it can be seen that initially when the hop count is less the number of time the logging takes place in the existing as well as the proposed method is somewhat same with the efficiency of more than $80 \%$, but as the hop count increases the $\%$ increase of the use of hash table in the existing method is more as compared to the proposed method. Moreover the efficiency $(€)$ of the existing method varies in between the range of 60 to 70 but in the proposed method it varies in between the range 80 to 90 .

The Figure 4 shows the number of hops with respect to the number of times the logging of the packet takes place. The $\mathrm{x}$-axis shows the number of hops and the $\mathrm{y}$-axis shows the number of time the hash table is referred. The Figure 5 shows the efficiency with respect to the number of hops for the existing and the proposed method. The $\mathrm{x}$-axis depicts the number of hops and the $\mathrm{y}$-axis depicts the efficiency in \%. 


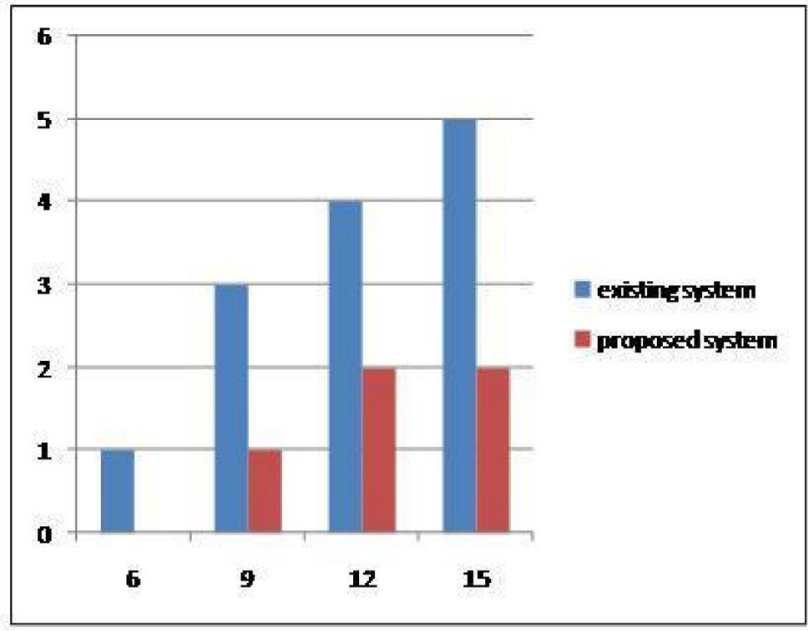

Figure 4. Comparison of number of referrals.

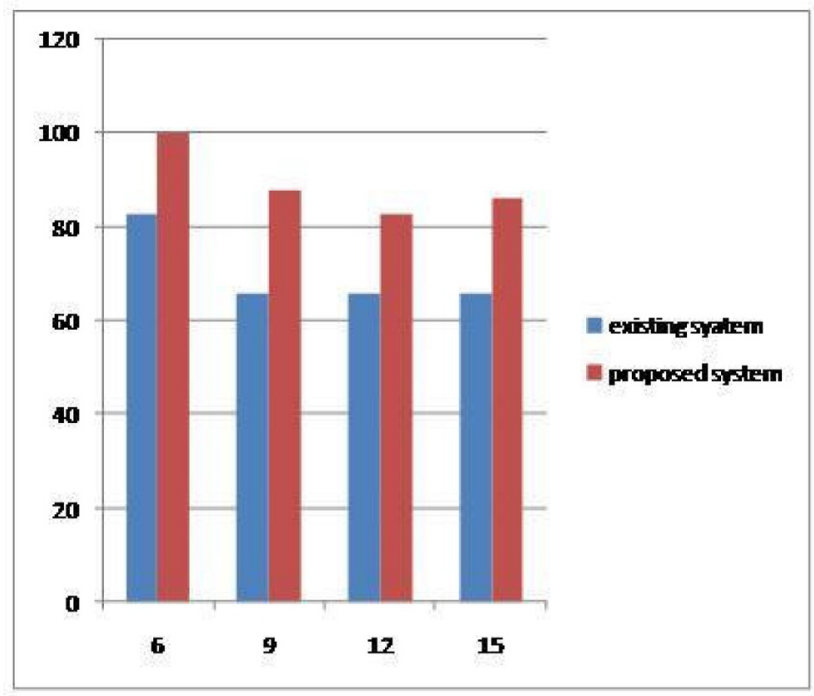

Figure 5. Comparison of efficiency.

\section{Conclusion}

In this paper, a new hybrid IP trace back method is proposed which uses a 16-bit marking field which efficiently traces back the origin of a network packet. The proposed method has been implemented and observed that it is more efficient than the existing method using 8-bit marking field. The number of reference to the hash table reduces greatly as compared with the existing method, thus having $20 \%$ more efficiency $(€)$. Also, this method provides a zero false positive and false negative rate in path reconstruction, and also, the amount of storage space required is very less compared to other existing trace back methods.

\section{References}

1. Liu W. Research on DoS attack and detection programming. Third International Symposium on Intelligent Information Technology Application. 2009.

2. Wang H, Jin C, Shin KG. Defense against spoofed IP traffic using hop-count filtering. IEEE/ACM Transactions on Networking, 2007; 15(1):40-53.

3. Al-Duwairi B, Govindarasu M. Novel hybrid schemes employing packet marking and logging for IP traceback. IEEE Transactions on Parallel and Distributed Systems. 2006; 17(5):403-18.

4. Gong C, Sarac K. IP traceback based on packet marking and logging. ICC 2005 IEEE International Conference on Communications. 2005; 2:1043-104.

5. Ansari NB. IP traceback with deterministic packet marking. IEEE Communication Letter. 2003; 7(4):162-64.

6. Snoeren C, Partridge C, Sanchez LA, Jones CE, Tchakountio F, Schwartz B, Kent ST, Strayer WT. Single-packet IP traceback. IEEE/ACM Transactions on Networking. 2002; 10(6):721-34.

7. Gong, SK. Toward a practical packet marking approach for IP traceback. Int J Netw Secur. 2009; 8(3):271-81.

8. Malliga S, Tamilarasi A. A hybrid scheme using packet marking and logging for IP traceback. International Journal of Internet Protocol Technology.2010; 5(1/2):81-91.

9. Choi KH, Dai HK. A marking scheme using Huffman codes for IP traceback. In the Proceedings of $7^{\text {th }}$ International Symposium on Parallel Architectures, Algorithms Networks (SPAN'04); 2004; Hong Kong, China. p. 421-28.

10. Yang M-H, Yang M-C. RIHT: A novel hybrid IP traceback Scheme. IEEE Transactions on Information Forensics and Security. 2012; 7(2).

11. Stocia I, Zhang H. Providing guaranteed services without peer flow management. Proceedings of ACM SIGCOMM'99, Boston, MA. p. 81-94.

12. Johnand W, Tafvelin S. Analysis of internet backbone traffic and header anomalies observed. Proceedings of IMC '07: 7th ACM SIGCOMM Conference on Internet Measurement; 2007; San Diego, CA. p. 111-16.

13. John W, Olovsson T. Detection of malicious traffic on backbone links via packet header analysis. Campus-wide Information Sys. 2008; 25(5):342-58. 